\title{
A new basic effect in retarding potential analyzers
}

\author{
Juan R. Sanmartín ${ }^{\text {a) }}$ and O. López-Rebollal \\ Escuela Técnica Superior de Ingenieros Aeronáuticos, Universidad Politécnica de Madrid, \\ 28040 Madrid, Spain
}

(Received 25 July 2000; accepted 2 August 2000)

\begin{abstract}
The Retarding Potential Analyzer (RPA) is the standard instrument for in situ measurement of ion temperature and other ionospheric parameters. The fraction of incoming ions rejected by a RPA produces perturbations that reach well ahead of a thin Debye sheath, a feature common to all collisionless, hypersonic flows past ion-rejecting bodies. This phenomenon is here found to result in a correction to Whipple's classical law for the current characteristic of an ideal RPA (sheath thin; inverse ram ion Mach number $M^{-1}$, and ram angle of RPA aperture $\theta$, small or moderately small). The current correction increases with the temperature ratio $T_{e} / T_{i}$, and ranges from a $15 \%-30 \%$ reduction at $M \theta=0$ to a $15 \%-30 \%$ increase at $M \theta=2$, for typical values of $M, T_{e} / T_{i}$ and transparency of aperture grid. Linear analysis of the perturbed plasma beyond the sheath rests on the fact that a Maxwellian undisturbed ion distribution is Vlasov-stable against quasineutralionacoustic waves. (C) 2000 American Institute of Physics. [S1070-664X(00)04511-0]
\end{abstract}

\section{INTRODUCTION}

Positively biased satellites such as the one used for electron collection by the Tethered Satellite System, flown by the National Aeronautics and Space Administration in February 1996 (TSS-1R mission), produce perturbations that spread beyond thin Debye sheaths. ${ }^{1}$ This phenomenon, which had been noticed both in experiments and in numerical calculations, ${ }^{2,3}$ appears to be a fundamental feature of collisionless, hypersonic plasma flows past ion-rejecting bodies: A low ion-density wake develops behind the body; ions missing from the wake are those perturbing the plasma far ahead. We argue here that this same phenomenon may affect the workings of a Retarding Potential Analyzer in a basic way.

Accurate values of the temperature $T_{i}$ of ionospheric ions are needed for establishing a valid energy budget of the upper atmosphere for the Earth, as well as for other planets, and may require processing data from a large number of measurements. ${ }^{4}$ In a laboratory plasma $T_{i}$ is a parameter hard to measure. The Retarding Potential Analyzer (RPA) has been used on board satellites since the beginning of the space age, having been kept as the standard in situ probe for determining $T_{i}$ in the ionosphere, ${ }^{5}$ a RPA will soon be flown on the Chinese ROCSAT- 1 spacecraft. ${ }^{6}$ RPA instruments have been also used on board rockets at the bottom of the ionosphere; ${ }^{7}$ on the Shuttle for measuring the plasma perturbations produced by the Shuttle itself; ${ }^{8,9}$ in the Venus ${ }^{4,10}$ and Mars $^{11,12}$ ionospheres; and on the TSS-1R electron collector. ${ }^{1}$

A RPA is a multigrid electrostatic probe. An entrance grid in the spacecraft wall is biased negative relative to the potential in the undisturbed plasma to repel, like the wall itself, incoming electrons; ions are collected by an electrode at the back of the instrument. Between the aperture and col-

a)Electronic mail: jrs@faia.upm.es lector there is a retarding grid biased at a positive value $V_{P}$, which rejects the less energetic ions. The collected ion current $I$ is registered as a function of $V_{P}$ as this potential is swept through a broad range of values. For a planar RPA, $T_{i}$ is determined by fitting the full experimental characteristic $I\left(V_{P}\right)$ to a formula derived by Whipple. ${ }^{13}$ Multigrid probes (Ion Traps) may also serve, however, to determine ion density and composition, drifts, and, by inference, electric fields in the ionosphere. ${ }^{14}$

Analysis of RPA collection in the Earth's ionosphere is simplified by the ordering of characteristic lengths: mean free path $\left(>10^{4}-10^{5} \mathrm{~cm}\right)$, ion thermal gyroradius $(\sim 3$ $\left.\times 10^{2} \mathrm{~cm}\right)$, and satellite size $\left(>10^{2} \mathrm{~cm}\right)$ are large compared to the aperture width $(\sim 10 \mathrm{~cm})$, which is itself large compared to the distance between grids $(<1 \mathrm{~cm})$, and the electron Debye length $\lambda_{\text {De }}(\sim 1 \mathrm{~cm})$. Outside a sheath of thickness $\lambda_{\mathrm{De}}$ next to the satellite wall and RPA aperture the plasma is quasineutral. Also, the spacecraft velocity is large compared with the ion thermal speed. Whipple then took an one-dimensional approach to derive a formula for the current reaching the collector. Whipple's law is actually used with instruments other than, though derived from, the RPA. ${ }^{9}$

Early discrepancies between data from planar RPAs and other measurements led to careful criticism of Whipple's ideal law for the characteristic $I\left(V_{P}\right)$. It was found that several effects inside the instrument (nonuniformity of potential in grid planes, energy-dependent grid transparencies, space charge between grids, limited grid width) could affect the characteristic. ${ }^{15}$ Instrument design was then improved to avoid problems in using the ideal RPA law for data interpretation. ${ }^{10,12}$ The effects of grid-mesh size and relative alignment on electron motion were analyzed very recently. ${ }^{16}$

The current characteristic may also be affected by conditions outside the RPA. Although Whipple's onedimensional approach rests on a condition of planar sheath, i.e., small $\lambda_{\text {De }},{ }^{17}$ RPAs have been used in the Solar Wind, where the Debye length is large; ${ }^{12}$ nonplanar sheaths make 
data analysis difficult, however. ${ }^{17,18}$ Whipple's law is also favored by high values of ion Mach number $M$ of the spacecraft motion through the plasma; ${ }^{19}$ too low a value of $M$ results in "internal shadowing," as in the case of limited grid width. ${ }^{8,19}$ Similarly, the law requires not too large an angle $\theta$ between normal to RPA sensor and spacecraft velocity; ${ }^{10}$ spacecraft spin keeps the angle varying, but measurements with moderately small $\theta$ are quite usual. ${ }^{10,12,20}$

The phenomenon studied here, however, affects Whipple's law in a more basic way in the sense that it holds for an ideal RPA, too. It was noted that ions rejected by the retarding grid should emerge from the RPA to form a jet with the same cross section of the entrance grid. ${ }^{21}$ Unless the transparency $\alpha_{\mathrm{E}}$ of that grid is very small, the field disturbance will not be confined to a sheath and will have a radically three-dimensional character; this will affect incoming ions and thus the collected current $I$. It was independently suggested that ions somehow reflected from the satellite wall could excite electrostatic Lower Hybrid waves, which might explain anomalous data in RPA experiments with spacecraft velocity near parallel to the geomagnetic field. ${ }^{20}$ Lower Hybrid waves were supposedly excited by the TSS-1R electron collector, too. ${ }^{22}$

In Sec. II we recall conditions leading to Whipple's ideal law, which appears as the $\alpha_{E}=0$ limit of a more general solution. An analysis of the perturbation beyond the RPA sheath at moderately small $\alpha_{E}$ is presented in Sec. III. A correction to Whipple's law is then derived in Sec. IV and results are discussed in Sec. V. In the Appendix our analysis is shown to rest on the condition that the undisturbed plasma be stable in the Vlasov sense.

\section{THE WHIPPLE MODEL}

We use a frame moving with the spacecraft and let the satellite wall be the (infinite) plane $x=0$, the entrance grid $E$ being a circle of radius $R$ centered at the origin; the plasma fills the half-space $x<0$ (Fig. 1). Behind the retarding grid $P$ there is a suppressor $G$ that is biased highly negative to turn back all electrons able to get past $E$, and to inhibit photo and secondary emission from the collector $C$. For simplicity we take equal satellite and grid- $E$ bias, $V_{E}<0$. Potential $V(\bar{r})$ and ion distribution function $f(\bar{r}, \bar{v})$ obey Poisson and steady Vlasov equations, respectively,

$$
\begin{aligned}
& \nabla^{2} V=4 \pi e\left[N_{\infty} \exp \left(\frac{e V}{k_{B} T_{e}}\right)-\int f d \bar{v}\right], \quad x<0, \\
& \bar{v} \cdot \nabla f-\frac{e}{m_{i}} \nabla V \cdot \frac{\partial f}{\partial \bar{v}}=0, \quad x<0,
\end{aligned}
$$

where $m_{i}$ is ion mass, $T_{e}$ electron temperature, and $N_{\infty}$ unperturbed ion or electron density. With $m_{e} U^{2} / k_{B} T_{e}$ very small and $e\left|V_{E}\right| / k_{B} T_{e}$ moderately large, electrons do follow Boltzmann's law except near the wall and grid $E$, where the electron density will be exponentially small anyway.

We partition $f$ in the form

$$
f \equiv f^{+}+f^{-}, \quad f^{+}\left(v_{x}<0\right) \equiv 0, \quad f^{-}\left(v_{x}>0\right) \equiv 0 .
$$

The boundary conditions are
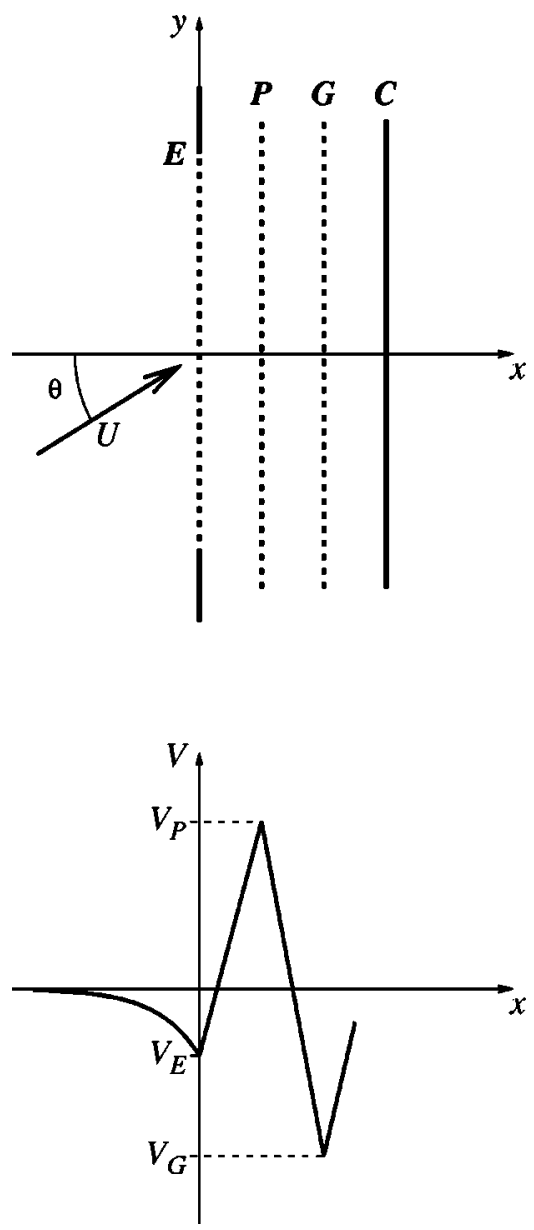

FIG. 1. Grid schematics and ideal potential profile of a Retarding Potential Analyzer (RPA); $E, P$, and $G$ are Entrance, Retarding and Suppressor grids, $C$ is the collector.

$$
\begin{aligned}
& V \rightarrow 0, \\
& f^{+}\left(v_{x}>0\right) \rightarrow f_{\infty}(\bar{v}) \quad \text { as } x \rightarrow-\infty, \\
& V=V_{E}, \\
& f^{-}\left(v_{x}<0\right)=H\left(R-r_{\perp}\right) g\left(\bar{r}_{\perp}, \bar{v}\right) \quad \text { at } x=0 .
\end{aligned}
$$

Here $f_{\infty}$ is the Maxwellian of a single ion species drifting at velocity $\bar{U}$,

$$
\begin{aligned}
f_{\infty}(\bar{v}) & =\frac{N_{\infty} m_{i}^{3 / 2}}{\left(2 \pi k_{B} T_{i}\right)^{3 / 2}} \exp \left[-\frac{\left(v_{x}-U_{x}\right)^{2}+\left(\bar{v}_{\perp}-\bar{U}_{\perp}\right)^{2}}{2 k_{B} T_{i} / m_{i}}\right] \\
& \equiv f_{M}\left(v_{x}-U_{x}, \bar{v}_{\perp}-\bar{U}_{\perp}\right),
\end{aligned}
$$

$\bar{r}_{\perp}\left(\bar{v}_{\perp}\right)$ is the position (velocity) vector perpendicular to the $x$ axis; $H$ is Heaviside's step function, which takes into account that ions striking the wall at $r_{\perp}>R$ are neutralized; and the distribution $g$ depends on ion incidence and RPA model. We let the drift $\bar{U}$ make an angle $\theta$ with the normal to the wall in the $x-y$ plane, $U_{x}=U \cos \theta, \bar{U}_{\perp}=\overline{1}_{y} U \sin \theta$ (Fig. 1).

We take the Mach number $M \equiv \sqrt{m_{i} U^{2} / k_{B} T_{i}}$ moderately large. In low Earth orbit, $M$ ranges from 8 to 4 for $T_{i}$ $\sim 1600 \mathrm{~K}$ and $\mathrm{O}^{+}$and $\mathrm{He}^{+}$ions, respectively; for $\mathrm{H}^{+}$, one has $M=3$ at $T_{i} \sim 750 \mathrm{~K}$. We will also consider moderately small incidence angles, writing $\sin \theta \sim \theta, \cos \theta \sim 1$; we let $M \theta$ 
be formally arbitrary, however, the characteristic value for $v_{\perp}$ in (5) then being $\sqrt{k_{B} T_{i} / m_{i}}$ or $U \theta$, whichever is the largest. The domain in ion phase-space for which collisional and finite satellite-wall effects are important (corresponding to undisturbed distant ions moving away from grid $E$ ) need not be considered, those ions making a fraction of order $\exp \left[-\frac{1}{2} M^{2}\left(1-\theta^{2}\right)\right]$ of the entire population; we shall actually ignore terms of order $M^{-2}$.

For an ideal RPA, as discussed in the Introduction, the distribution $g$ in boundary condition (4b) takes a simple form. Since width-to-depth grid ratio and $M$ are moderately large, and $\theta$ is small, ions arriving at $E$ with $v_{x}$ such that $\frac{1}{2} m_{i} v_{x}^{2}<e\left(V_{P}-V_{E}\right)$, which are rejected by the retarding grid $P$, emerge from $E$ near the point of entry with the same $\bar{v}_{\perp}$ and opposite $v_{x}$. This leads to

$$
\begin{aligned}
g \approx & \alpha_{E}^{2} H\left[\sqrt{2 e \frac{V_{P}-V_{E}}{m_{i}}}-\left|v_{x}\right|\right] f^{+}\left(x=0, \bar{r}_{\perp}, \bar{v}_{\perp},\left|v_{x}\right|\right), \\
& v_{x}<0 .
\end{aligned}
$$

We consider $\alpha_{E}^{2}$ formally small, and write expansions $V=V_{0}+\alpha_{E}^{2} V_{1}+\ldots, f^{ \pm}=f_{0}^{ \pm}+\alpha_{E}^{2} f_{1}^{ \pm}+\ldots$, the effect of the rejected ions proving to be moderately small for actual values $\alpha_{E}^{2} \sim 1$. Since $g$ vanishes with $\alpha_{E}^{2}$, and $V_{E}$ is negative, we will have $f_{0}^{-} \equiv 0$. The solution of order zero in $\alpha_{E}^{2}$ is thus one-dimensional, Eqs. (1) and (2) reading

$$
\begin{aligned}
& \frac{d^{2} V_{0}}{d x^{2}}=4 \pi e\left[N_{\infty} \exp \left(\frac{e V_{0}}{k_{B} T_{e}}\right)-\int_{v_{x}>0} d \bar{v} f_{0}^{+}\right], \\
& v_{x} \frac{\partial f_{0}^{+}}{\partial x}-\frac{e}{m_{i}} \frac{d V_{0}}{d v_{x}} \frac{\partial f_{0}^{+}}{\partial v_{x}}=0, \quad v_{x}>0 .
\end{aligned}
$$

Equation (8) with boundary condition (3b) is trivially solved, giving

$$
\begin{aligned}
& f_{0}^{+}=f_{M}\left[\sqrt{v_{x}^{2}+\frac{2 e}{m_{i}} V_{0}(x)}-U_{x}, \bar{v}_{\perp}-\bar{U}_{\perp}\right], \\
& v_{x}>\sqrt{-\frac{2 e}{m_{i}} V_{0}(x)>0,} \\
& \int d \bar{v} f_{0}^{+} \approx N_{\infty}\left(1+e V_{0} / k T_{i} M^{2}\right) .
\end{aligned}
$$

Using (10) in (7) to integrate once with boundary condition (3a) yields

$$
\frac{\lambda_{\mathrm{De}}^{2}}{2}\left[\frac{d}{d x}\left(\frac{e V_{0}}{k T_{e}}\right)\right]^{2}=\exp \left(\frac{e V_{0}}{k T_{e}}\right)-1-\frac{e V_{0}}{k T_{e}}-\frac{T_{e}}{2 M^{2} T_{i}}\left(\frac{e V_{0}}{k T_{e}}\right)^{2},
$$

$V_{0}(0)=V_{E}$

Equation $\left(7^{\prime}\right)$ can be readily integrated to determine $V_{0}(x) / V_{E}$ as function of $x / \lambda_{\text {De }}$ and parameters $e V_{E} / k_{B} T_{e}$ and $M^{2} T_{i} / T_{e}$; here, $\lambda_{\mathrm{De}}$ is the Debye length, $\sqrt{4 \pi e^{2} N_{\infty} / k_{B} T_{e}}$. At large $|x| / \lambda_{\text {De }}, V_{0}(x) / V_{E}$ vanishes as $\exp \left(x / \lambda_{\text {De }}\right)$ and $f_{0}^{+}$approaches $f_{\infty}(\bar{v})$.
Since the particle flux along $x$ is conserved, the lowest order current to the collector is due to ions with velocity $v_{x}$ outside the sheath such that $\frac{1}{2} m_{i} v_{x}^{2}>e V_{P}$,

$$
\begin{aligned}
\frac{I_{0}}{\alpha} & =A_{E} e \int d \bar{v}_{\perp} \int_{\sqrt{2 e V_{P} / m_{i}}}^{\infty} v_{x} d v_{x} f_{\infty}(\bar{v}) \\
& =A_{E} e N_{\infty} U \cos \theta\left[\frac{1-\operatorname{erf} \Delta}{2}+\sqrt{\frac{2}{\pi}} \frac{e^{-\Delta^{2}}}{2 M \cos \theta}\right],
\end{aligned}
$$

$\alpha=\alpha_{E} \times \alpha_{P} \times \alpha_{G}$ being the overall RPA transparency (Fig. 1) and

$$
\Delta \equiv \sqrt{\frac{e V_{P}}{k_{B} T_{i}}}-\frac{M \cos \theta}{\sqrt{2}} .
$$

This is Whipple's formula. ${ }^{13}$ As $V_{P}$ is increased, the bracket in (11) decreases from 1 to 0 , the decrease being centered around $\Delta=0$, or $e V_{P}=\frac{1}{2} m_{i} U^{2} \cos ^{2} \theta$. When limited to this lowest order perturbation, no small- $\theta$ approximation is used in the solution, so as to recover Whipple's result as usually written.

\section{LOW ENTRANCE TRANSPARENCY PERTURBATIONS}

Terms of order $\alpha_{E}^{2}$ lead to a correction to Whipple's formula. Inside the sheath, the equation for $f_{1}^{-}$in (2) is

$$
\left(v_{x} \frac{\partial}{\partial x}+\bar{v}_{\perp} \cdot \frac{\partial}{\partial \bar{r}_{\perp}}\right) f_{1}^{-}-\frac{e}{m_{i}} \frac{d V_{0}}{d x} \frac{\partial f_{1}^{-}}{\partial v_{x}}=0, \quad v_{x}<0,
$$

which must be solved with boundary condition (4b). Using $f_{0}^{+}$for $g$ to first order in Eq. (6), the second term in the parenthesis of (13) is of order $\lambda_{\mathrm{De}} / M R\left(\theta \lambda_{\mathrm{De}} / R\right)$ relative to the first, for small (large) $M \theta$, and may be dropped. The solution to (13) is then readily determined; as $x / \lambda_{\mathrm{De}} \rightarrow-\infty$ one finds

$$
\begin{aligned}
f_{1}^{-}\left(\frac{x}{\lambda_{\mathrm{De}}} \rightarrow-\infty, \bar{r}_{\perp}\right)= & H\left(R-r_{\perp}\right) H\left(\sqrt{\frac{2 e V_{P}}{m_{i}}}-\left|v_{x}\right|\right) \\
& \times f_{M}\left(v_{x}+U_{x}, \bar{v}_{\perp}-\bar{U}_{\perp}\right) .
\end{aligned}
$$

In the region outside the sheath, the equation for $f_{1}^{-}$is

$$
\left(v_{x} \frac{\partial}{\partial x}+\bar{v}_{\perp} \cdot \frac{\partial}{\partial \bar{r}_{\perp}}\right) f_{1}^{-}=0
$$

thermal motion spreading the jet of rejected ions over distances of order $M R$; if $M \theta$ is large there is an imbedded subregion with caracteristic length $R / \theta$ along $x$. Equation (15) is solved by Fourier transforming with respect to $\bar{r}_{\perp}$, and then integrating with respect to $x$, to yield

$$
\begin{aligned}
\widetilde{f}_{1}^{-}\left(x, \bar{k}_{\perp}\right) & \equiv \int d \bar{r}_{\perp} f_{1}^{-}\left(x, \bar{r}_{\perp}\right) \exp \left(-i \bar{k}_{\perp} \cdot \bar{r}_{\perp}\right) \\
& =\widetilde{f}_{1}^{-}\left(0, \bar{k}_{\perp}\right) \exp \left(-i \frac{\bar{k}_{\perp} \cdot \bar{v}_{\perp}}{v_{x}} x\right),
\end{aligned}
$$


with the $\bar{r}_{\perp}$ integration extended to the entire $y-z$ plane. Here, $\tilde{f}_{1}^{-}\left(0, \bar{k}_{\perp}\right)$ must equal the Fourier transform of $f_{1}^{-}\left(x / \lambda_{\mathrm{De}} \rightarrow-\infty, \bar{r}_{\perp}\right)$ above,

$$
\begin{aligned}
\tilde{f}_{1}^{-}\left(0, \bar{k}_{\perp}\right)= & \frac{2 \pi R}{k_{\perp}} J_{1}\left(k_{\perp} R\right) H\left[\sqrt{\frac{2 e V_{P}}{m_{i}}}-\left|v_{x}\right|\right] f_{M}\left(v_{x}\right. \\
& \left.+U_{x}, \bar{v}_{\perp}-\bar{U}_{\perp}\right), \quad v_{x}<0,
\end{aligned}
$$

where $J_{1}$ is the Bessel function of first kind and order. Integration of $\widetilde{f}_{1}^{-}$in (16a) over $\bar{v}$ yields $\widetilde{N}_{1}^{-}\left(x, \bar{k}_{\perp}\right)$, and Fourier inversion with respect to $\bar{k}_{\perp}$ yields $N_{1}^{-}(\bar{r})$ outside the sheath. In particular, one finds

$$
\begin{aligned}
& N_{1}^{-}\left(x=0, \bar{r}_{\perp}\right)=N_{\infty} \frac{1+\operatorname{erf} \Delta}{2} H\left(R-r_{\perp}\right), \\
& N_{1}^{-}\left(|x| \ll M R, \bar{r}_{\perp}\right) \approx N_{\infty} \frac{1+\operatorname{erf} \Delta}{2} H\left(R-\left|\bar{r}_{\perp}+\theta x \overline{1}_{y}\right|\right), \\
& N_{1}^{-}(|x| \gg M R, z=0) \approx N_{\infty} \frac{1+\operatorname{erf} \Delta}{2} \times \frac{1}{2}\left(\frac{M R}{x}\right)^{2} \\
& \quad \times \exp \left(-\frac{M^{2}}{2}\left(\theta+\frac{y}{x}\right)^{2}\right) .
\end{aligned}
$$

Equation (18a) reduces to (17) unless $M \theta$ is large. In both illustrative results (18a), and (18b) we ignored terms of order $1 / M$.

The density $N_{1}^{-}$gives rise to a first-order potential perturbation, $V_{1}$, which in term produces a first-order perturbation of the incoming ion population, $f_{1}^{+}$. Outside the sheath, where quasineutrality holds, Eq. (1) to first order reads

$$
N_{\infty} \frac{e V_{1}}{k_{B} T_{e}} \approx \int f_{1}^{+} d \bar{v}+N_{1}^{-},
$$

while the equation for $f_{1}^{+}$in (2) takes the form

$$
\begin{aligned}
& \left(v_{x} \frac{\partial}{\partial x}+\bar{v}_{\perp} \cdot \frac{\partial}{\partial \bar{r}_{\perp}}\right) f_{1}^{+} \\
& \quad=\frac{e}{m_{i}}\left(\frac{\partial V_{1}}{\partial x} \frac{\partial}{\partial v_{x}}+\frac{\partial V_{1}}{\partial \bar{r}_{\perp}} \cdot \frac{\partial}{\partial \bar{v}_{\perp}}\right) f_{\infty}, \quad v_{x}>0 .
\end{aligned}
$$

Fourier transforming (20) and using boundary condition (3b) to first order, one finds

$$
\begin{aligned}
\widetilde{f}_{1}^{+}\left(x, \bar{k}_{\perp}\right)= & \int_{-\infty}^{x} \frac{d x^{\prime}}{v_{x}} \exp \left[-i \frac{\bar{k}_{\perp} \cdot \bar{v}_{\perp}}{v_{x}}\left(x-x^{\prime}\right)\right] \\
& \times \frac{e}{m_{i}}\left\{\frac{\partial \widetilde{V}_{1}\left(x^{\prime}, \bar{k}_{\perp}\right)}{\partial x^{\prime}} \frac{\partial}{\partial v_{x}}\right. \\
& \left.+\widetilde{V}_{1}\left(x^{\prime}, \bar{k}_{\perp}\right) i \bar{k}_{\perp} \cdot \frac{\partial}{\partial \bar{v}_{\perp}}\right\} f_{\infty},
\end{aligned}
$$

and then, integrating over $\bar{v}$, one arrives at $\widetilde{N}_{1}^{+}$in terms of $\widetilde{V}_{1}$,

$$
\begin{aligned}
& \frac{\tilde{N}_{1}^{+}\left(x, \bar{k}_{\perp}\right)}{N_{\infty}}=\int_{-\infty}^{x} d x^{\prime}\left\{L_{x}\left[\left(x-x^{\prime}\right) \bar{k}_{\perp}\right] \frac{\partial}{\partial x^{\prime}}\right. \\
& \left.+i \bar{k}_{\perp} \cdot \bar{L}_{\perp}\left[\left(x-x^{\prime}\right) \bar{k}_{\perp}\right]\right\} \frac{e \widetilde{V}_{1}\left(x^{\prime}, \bar{k}_{\perp}\right)}{k_{B} T_{i}}, \\
& \bar{L}\left[\left(x-x^{\prime}\right) \bar{k}_{\perp}\right] \equiv \\
& \quad \frac{k_{B} T_{i}}{N_{\infty} m_{i}} \int_{v_{x}>0} \frac{d \bar{v}}{v_{x}} \\
& \quad \times \exp \left[-i\left(x-x^{\prime}\right) \frac{\bar{k}_{\perp} \cdot \bar{v}_{\perp}}{v_{x}}\right] \frac{\partial f_{\infty}}{\partial \bar{v}} .
\end{aligned}
$$

Finally, Fourier transforming Eq. (19) one obtains an equation for $\widetilde{V}_{1}\left(x, \bar{k}_{\perp}\right)$

$$
\beta \frac{e \widetilde{V}_{1}}{k_{B} T_{i}}-\frac{\tilde{N}_{1}^{+}}{N_{\infty}}=\frac{\tilde{N}_{1}^{-}}{N_{\infty}}, \quad \beta \equiv \frac{T_{i}}{T_{e}}, \quad x<0 .
$$

Equation (24) is a linear integral equation defined in the half-space $x<0$; singular; and with a difference kernel as in the Wiener-Hopf problem. ${ }^{23}$ Our equation, however, is of Volterra type. In order to solve it, we consider an extended problem: Use Eq. (24) with the function $\widetilde{N}_{1}^{-}(x<0)$ on the right-hand-side continued to the halfspace $x>0$, to find $\widetilde{V}_{1}\left(x, \bar{k}_{\perp}\right)$ in the entire range $-\infty<x<\infty$. We must then show that our choice of right-hand-side in (24) for $x>0$ has no effect on the resulting solution for $\widetilde{V}_{1}$ in the physical domain of definition $(x<0)$; this is proved in the Appendix. Here, by taking Fourier transforms with respect to $x$, e.g.,

$$
\widetilde{\widetilde{V}}_{1}\left(k_{x}, \bar{k}_{\perp}\right) \equiv \int_{-\infty}^{\infty} d x \exp \left(-i k_{x} x\right) \widetilde{V}_{1}\left(x, \bar{k}_{\perp}\right),
$$

one uses Eq. (24) to solve for $\widetilde{\widetilde{V}}_{1}$ and then transform back with respect to $k_{x}$ to find

$$
\begin{aligned}
& \frac{e \widetilde{V}_{1}\left(x, \bar{k}_{\perp}\right)}{k_{B} T_{i}}=\int_{-\infty}^{\infty} \frac{d k_{x}}{2 \pi} \frac{\exp \left(i k_{x} x\right)}{\beta+L(\bar{k})} \frac{\widetilde{N}_{1}^{-}(\bar{k})}{N_{\infty}}, \\
& L(\bar{k})=-\int_{0}^{\infty} d s \exp \left(-i k_{x} s\right) i \bar{k} \cdot \bar{L}\left[s \bar{k}_{\perp}\right] .
\end{aligned}
$$

Using (23) in Eq. (26) for $L(\bar{k})$, the $\bar{v}_{\perp}$ integration can be carried out exactly; also, changing variable from $s$ to $\sigma$ $\equiv \sqrt{k T_{i} / m_{i}} \times k_{\perp} s / v_{x} \sqrt{2}$ the $v_{x}$ integral can be carried out, too, when terms exponentially small are neglected. We then find $L(\bar{k})$ in terms of a single integral

$$
\begin{gathered}
L(\bar{k}) \equiv L[\zeta(\bar{k})] \equiv \int_{0}^{\infty} 2 \sigma d \sigma \exp \left(-\sigma^{2}\right) \exp (2 i \sigma \zeta) \\
\zeta \equiv \frac{-\bar{k} \cdot \bar{U}}{k_{\perp} \sqrt{2 k_{B} T_{i}}},
\end{gathered}
$$

where we now neglected terms of order $M^{-2}$; note that perturbations steady in the satellite frame have frequency $-\bar{k} \cdot \bar{U}$ in the ionospheric frame. Next, using (16a), and (16b) in 


$$
\tilde{N}_{1}^{-}(\bar{k})=\int_{-\infty}^{\infty} d x \exp \left(-i k_{x} x\right) \int_{v_{x}<0} d \bar{v} \widetilde{f}_{1}^{-}\left(x, \bar{k}_{\perp}\right)
$$

the $\bar{v}_{\perp}$ and $x$ integrations can be carried out exactly, while the $v_{x}$ integral can be explicitly evaluated to order $M^{-2}$

$$
\begin{aligned}
\frac{\tilde{N}_{1}^{-}\left(\bar{k}_{\perp}, \zeta\right)}{N_{\infty}} \approx & \sqrt{2 \pi^{3}} \frac{M R J_{1}\left(k_{\perp} R\right)}{k_{\perp}^{2}} \exp \left[-(\zeta+\sqrt{2} M \theta \sin \phi)^{2}\right] \\
& \times\left[1+\operatorname{erf} \Delta-\sqrt{\frac{2}{\pi}} \frac{e^{-\Delta^{2}}}{M}\{1-2\right. \\
& \times(\zeta+\sqrt{2} M \theta \sin \phi)(\zeta+\sqrt{1 / 2} M \theta \sin \phi)\}]
\end{aligned}
$$

where we used polar coordinates $k_{\perp}, \phi$ for $\bar{k}_{\perp}$ with $\sin \phi$ $\equiv k_{y} / k_{\perp}$, and changed from $k_{x}$ to $\zeta$. We thus finally obtain

$$
\begin{aligned}
\frac{e \widetilde{V}_{1}\left(x, \bar{k}_{\perp}\right)}{k T_{i}}= & \int_{-\infty}^{\infty} \frac{d \zeta}{\beta+L(\zeta)} \exp \left[-i \frac{k_{\perp} x}{M}(\zeta \sqrt{2}+M \theta \sin \phi)\right] \\
& \times \frac{k_{\perp} \widetilde{N}_{1}^{-}\left(\bar{k}_{\perp}, \zeta\right)}{M \pi \sqrt{2} N_{\infty}} .
\end{aligned}
$$

\section{MODIFIED CURRENT LAW}

Positive $v_{x}$ ions entering the sheath within the cylinder $r_{\perp}<R$ with energy $\frac{1}{2} m_{i} v_{x}^{2}>\frac{1}{2} m_{i} v_{x \min }{ }^{2} \equiv e\left(V_{P}-\alpha_{E}^{2} V^{*}\right)$ will cross the retarding grid $P$ and reach the collector (Fig. 1); here, $V^{*}$ is $V_{1}\left(-x \ll M R, R / \theta ; \bar{r}_{\perp}\right)$. Within the sheath, the ion flux through the lateral surface of that cylinder leads to corrections of order $\lambda_{\mathrm{De}} / M R$ or $\theta \lambda_{\mathrm{De}} / R$, which we neglect. To first order in $\alpha_{E}^{2}$ the current collected is thus given by

$$
\begin{aligned}
I & =\alpha e \int_{A_{E}} d \bar{r}_{\perp} \int d \bar{v}_{\perp} \int_{v_{x} \min }^{\infty} v_{x} d v_{x}\left(f_{\infty}+\alpha_{E}^{2} f^{*}\right) \\
& \approx I_{0}+\alpha_{E}^{2} I_{1}
\end{aligned}
$$

with $f^{*} \equiv f_{1}^{+}\left(-x \ll M R, R / \theta, \bar{r}_{\perp}\right)$. The integral involving $f_{\infty}$ yields Whipple's result, $I_{0}$, plus a small term due to the perturbed potential $V^{*}$ in $v_{x \min }$. This term is added to the integral involving $f^{*}$, where we may set $v_{x \text { min }}$ $\approx \sqrt{2 e V_{P} / m_{i}}$, to get the full current correction, $\alpha_{E}^{2} I_{1}$

$$
\begin{aligned}
I_{1}= & \alpha e \int_{A_{E}} d \bar{r}_{\perp} \int d \bar{v}_{\perp}\left[\frac{e V^{*}}{m_{i}} f_{\infty}\left(v_{x}=\sqrt{\frac{2 e V_{P}}{m_{i}}}\right)\right. \\
& \left.+\int_{\sqrt{2 e V_{P} / m_{i}}}^{\infty} v_{x} d v_{x} f^{*}\right] .
\end{aligned}
$$

We take $f^{*}$ from (21), where we set $x=0$, integrate by parts the first term within braces, and take the Fourier inverse with respect to $\bar{k}_{\perp}$. The full first order current then becomes

$$
\begin{aligned}
I_{1}= & \alpha e \int_{A_{E}} d \bar{r}_{\perp} \int d \bar{v}_{\perp} \int_{\sqrt{2 e V_{P} / m_{i}}}^{\infty} d v_{x} \int \frac{d \bar{k}_{\perp}}{4 \pi^{2}} \exp \left(i \bar{k}_{\perp} \cdot \bar{r}_{\perp}\right) \\
& \times \int_{-\infty}^{0} d x^{\prime} \exp \left(i \frac{\bar{k}_{\perp} \cdot \bar{v}_{\perp}}{v_{x}} x^{\prime}\right) \frac{e \widetilde{V}_{1}\left(x^{\prime}, \bar{k}_{\perp}\right)}{m_{i}} i \bar{k}_{\perp} \\
& \cdot\left(\frac{\partial}{\partial \bar{v}_{\perp}}-\frac{\bar{v}_{\perp}}{v_{x}} \frac{\partial}{\partial v_{x}}\right) f_{\infty},
\end{aligned}
$$

with $\widetilde{V}_{1}\left(x^{\prime}, \bar{k}_{\perp}\right)$ given by Eq. (30). The resulting multiple integral for $I_{1}$ can be considerably simplified. The $\bar{r}_{\perp}$ and $\bar{v}_{\perp}$ integrations are straightforward. Changing variable from $x^{\prime}$ to $\sigma \equiv-\sqrt{k T_{i} / m_{i}} \times k_{\perp} x^{\prime} / v_{x} \sqrt{2}$, the $k_{\perp}$ integral can be carried out exactly, and the $\sigma$ and $v_{x}$ integrations can be carried out to order $M^{-2}$ in terms of the $L(\zeta)$ function.

The final result is

$$
\begin{aligned}
I_{1}= & -\alpha A_{E} e N_{\infty} U \\
& \times\left[c_{1} \frac{1-\operatorname{erf}^{2} \Delta}{2}-c_{2} \frac{e^{-\Delta^{2}}}{M} \frac{1+\operatorname{erf} \Delta}{2}-c_{3} \frac{e^{-\Delta^{2}}}{M} \frac{1-\operatorname{erf} \Delta}{2}\right] .
\end{aligned}
$$

Here $c_{1}, c_{2}$, and $c_{3}$ are functions of $\beta \equiv T_{i} / T_{e}$ and $M \theta$, and are given by double integrals involving $L(\zeta)$

$c_{j}(\beta, M \theta)$

$$
=\int_{0}^{2 \pi} \frac{d \phi}{2 \pi} \int_{-\infty}^{\infty} \frac{d \zeta}{\sqrt{2 \pi}} \frac{\exp \left[-(\zeta+\sqrt{2} M \theta \sin \phi)^{2}\right]}{\beta+L(\zeta)} h_{j},
$$

$\sqrt{2} h_{1}=L(\zeta)$,

$\sqrt{\pi} h_{2}=1-\left(1-2 \zeta^{2}-\zeta \sqrt{2} M \theta \sin \phi\right) L(\zeta)$,

$\sqrt{\pi} h_{3}=[1-2(\zeta+\sqrt{2} M \theta \sin \phi)(\zeta+\sqrt{1 / 2} M \theta \sin \phi)] L(\zeta)$.

Figures 2-4 show $c_{j}, j=1-3$; they are real because the real and imaginary parts of $L$ are even and odd functions of $\zeta$, respectively.

We note that $c_{2}$ approaches some limit function $c_{2}(\beta, \infty)$ (whereas $c_{1}, c_{3}$ vanish) when formally taking $M \theta \rightarrow \infty$. That function is easily obtained by writing $\zeta$ $+\sqrt{ } 2 M \theta \sin \varphi \equiv \zeta^{\prime}=O(1)$, and using the asymptotic approximation for $L(\zeta)$ at large values of its argument, $-L(\zeta)$ $\sim 1 / 2 \zeta^{2} \sim 1 /\left(4 M^{2} \theta^{2} \sin ^{2} \varphi\right) \ll 1$ (see the Appendix). We then find

$$
\begin{aligned}
c_{2} \approx & \int_{0}^{2 \pi} \frac{d \phi}{2 \pi} \int_{-\infty}^{\infty} \frac{d \zeta^{\prime}}{\sqrt{2 \pi}} \\
& \times \frac{\exp \left(-\zeta^{\prime 2}\right)}{\beta} \frac{1-\left(-2 \zeta^{2}+\zeta^{2}\right) \times\left(-1 / 2 \zeta^{2}\right)}{\sqrt{\pi}} \\
= & \frac{1}{2 \beta \sqrt{2 \pi}} .
\end{aligned}
$$

Values at $M \theta=2$ in Fig. 3 are already close to the asymptotic result (36). 


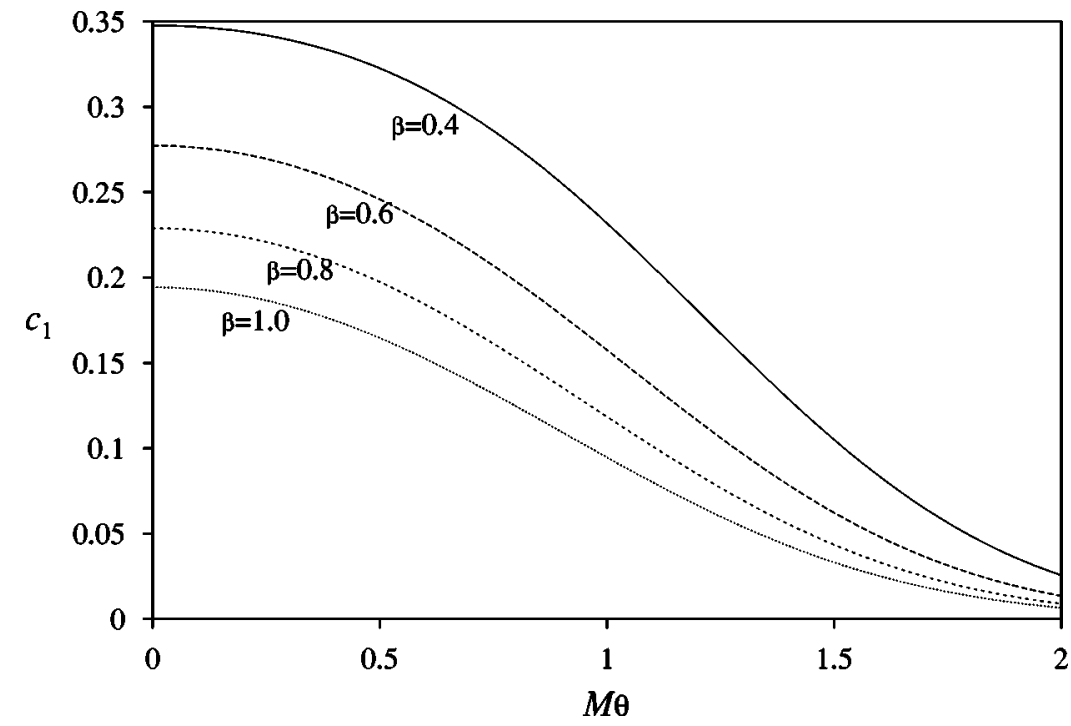

FIG. 2. Coefficient $c_{1}(\beta, M \theta)$ in Eq. (33) for the current correction; $\beta, M$, and $\theta$ are temperature ratio $T_{i} / T_{e}$, ram Mach number for ions, and ram angle for the RPA, respectively.

One might have surmized full vanishing of the correction $I_{1}$ as $M \theta \rightarrow \infty$, the reflected-ion density in Eq. (18b) being exponentially small $\left[N_{1}^{-} / N_{\infty} \sim \exp \left(-2 M^{2} \theta^{2}\right)\right]$ at the incidence angle $y / x \approx \theta$; the density maximum lies of course along the reflected velocity $-U_{x}, \bar{U}$, i.e., at $y / x=-\theta$ (Fig. $1)$. Further, with $\beta$ of order unity and $(\beta+$ Real part of $L)$ $>0$ throughout the integration in (25), $V_{1}$ behaves in a way similar to $N_{1}^{-}$. Ions incident on the RPA should then be negligibly perturbed at distances $|x| / M R \geqslant O(1)$. Note, however, that incident ions, and ions reflected from all grid$E$ points below each particular incidence point in Fig. 1, do cross over distances $|x| \sim R / \theta \ll M R$, where the density $N_{1}^{-}$ is given by (18a); this keeps $I_{1}$ from vanishing with $1 / M \theta$.

Our correction to Whipple's law is then the replacement

$$
I_{0} \rightarrow I_{0}+a_{E}^{2} I_{1},
$$

with $I_{0}$ and $I_{1}$ given by Eqs. (11) and (33), respectively. At $\Delta$ negative enough few ions are rejected by the RPA and the correction is negligible $\left(\alpha_{E}^{2} I_{1} / I_{0} \rightarrow 0\right.$ as $\left.\Delta \rightarrow-\infty\right)$. For $\Delta$ moderately positive the dependence of the ratio $\alpha_{E}^{2} I_{1} / I_{0}$ on all four parameters, $\Delta, M, \beta$, and $M \theta$, is quite complex.

At normal incidence $(M \theta=0)$ the correction ratio is negative, its magnitude increasing with increasing $M$ or decreasing $\beta$. The current reduction goes through a maximum somewhere between $\Delta=0$ and $\Delta=2$. At $\Delta=1$ in particular, and taking $\alpha_{E}^{2}=0.9$, current is reduced by $12 \%-20 \%$ at $\beta$ $=1$ and $M=4-8$; and by $20 \%-33 \%$ at $\beta=0.5$ and $M$ $=4-8$.

At $M \theta=2$, however, the correction ratio is positive; also, it grows monotonically with $\Delta$. Its magnitude now increases when either Mach number $M$ or temperature ratio $\beta$ decreases. At $\Delta=2$, and again taking $\alpha_{E}^{2}=0.9$, the corrected current is larger than Whipple's current by $11 \%$ at $\beta=1$, $M=8$, and by $33 \%$ at $\beta=0.5, M=4$.

The greater magnitude of our correction at lower $\beta$ is manifest in the first term of (24); at large electron temperature the electrons refuse to cooperate, so to speak, in balancing the reflected-ion density. On the other hand, the change

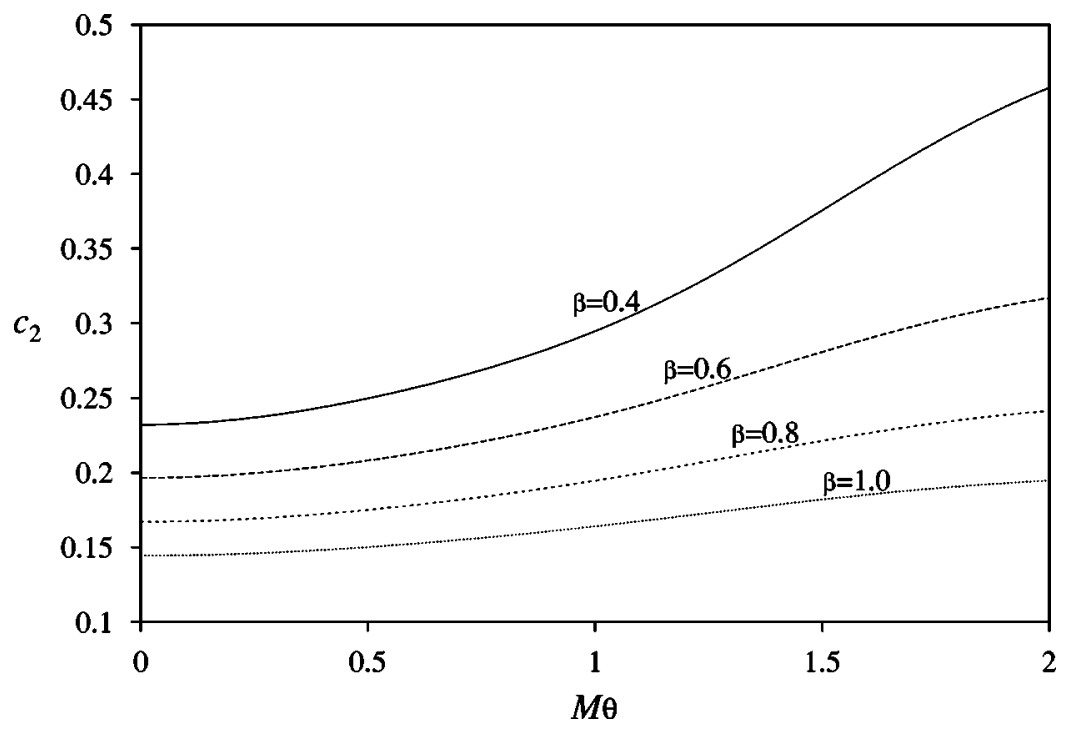

FIG. 3. Coefficient $c_{2}(\beta, M \theta)$ in Eq. (33). 


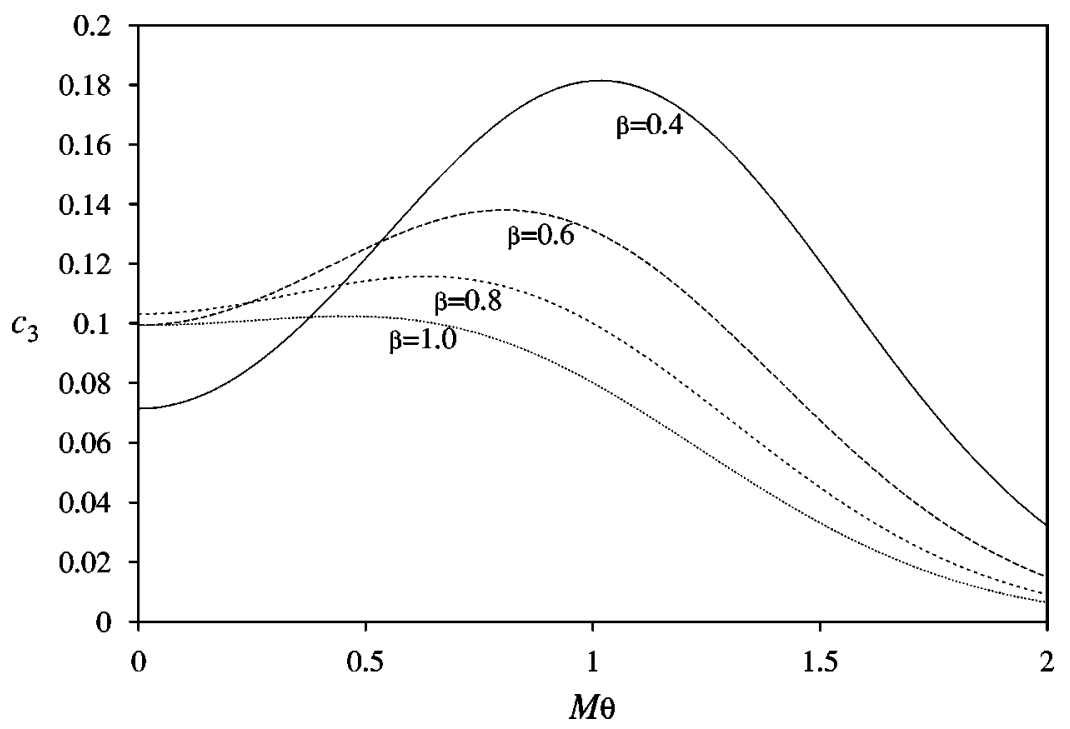

FIG. 4. Coefficient $c_{3}(\beta, M \theta)$ in Eq. (33).

of sign in $I_{1}$ as $M \theta$ increases is the result of two competing effects. Incoming ions are clearly subject to a retarding deceleration, because $V_{1}$, as $N_{1}^{-}$itself, is positive; the second term in the bracket of Eq. (32) should then be negative, whereas the first term is positive. One can easily show, in particular, that, at large $M \theta$ [when $c_{1}, c_{3}$ vanish in Eq. (33), yielding $\left.I_{1}>0\right]$, the integrations in (32) over its first and second terms yield $2 I_{1}$ and $-I_{1}$, respectively.

Our correction to the extremum of the slope $d I / V_{P}$ of the current characteristic is weaker than the correction for the current itself; the slope extremum is sometimes used for a simple estimate of ion temperature. Use of Whipple's current (11) yields

$$
-\frac{d I_{0}}{d V_{P}}=\frac{\alpha A_{E} e^{2} N_{\infty}}{\sqrt{2 \pi m_{i} k_{B} T_{i}}} \exp \left(-\Delta^{2}\right),
$$

with the extremum at $\Delta=0$ and $k T_{i} \propto 1 /\left|d I / d V_{P}\right|^{2}{ }_{\text {max }}$. For $M \theta=2$ in our full-current formula the extremum still occurs at $\Delta \approx 0$ but its value is reduced by a factor of $1-\alpha_{E}^{2}\left(c_{2}\right.$ $\left.-c_{3}\right) / M$; for $\alpha_{E}^{2}=0.9$ the corrected temperature is then smaller than Whipple's temperature by $4 \%$ at $\beta=1, M=8$ and by $15 \%$ at $\beta=0.5, M=4$. For $M \theta=0$ the extremum occurs at $\Delta_{\text {ext }} \approx-2 \alpha_{E}^{2} c_{1} / \sqrt{ } \pi$, with its value increased by a factor $1+\Delta_{\text {ext }}^{2}$; the actual temperature is now larger than Whipple's value by $7 \%$ at $\beta=1$ and by $15 \%$ at $\beta=0.5$.

\section{CONCLUSIONS}

We have described a basic correction to Whipple's classical law [Eqs. (11) and (12)] for the current $I$ to a planar Retarding Potential Analyzer (RPA), which is a standard multigrid probe serving to determine ion temperature $T_{i}$ and other ionospheric parameters. Grid-related effects inside the RPA, which could invalidate Whipple's law, had been corrected in the past by proper instrument design. The effect here considered arises from those ions that are rejected by the RPA and perturb the plasma far ahead of the sheath at its front. This phenomenon is a fundamental feature of collisionless, hypersonic plasma flows past ion-rejecting bodies, and was noticed in a broader context both in experiments and in numerical calculations. Ion rejection is a process essential to a RPA.

In deriving our correction to the current, we considered the case of a single ion species, which is easily generalized, and took the transparency of the aperture grid $\alpha_{E}$ formally small, the effect of the rejected ions proving to be moderately small for actual values $\alpha_{E} \sim 1$. We also considered an ideal RPA as implied in Whipple's law: We assumed inverse ion ram Mach number $M^{-1}$, ram angle of aperture normal $\theta$, and ratio of Debye length to aperture width, small or moderately small, but let $M \theta$ arbitrary. For a "nonideal" RPA, rejected ions would still affect the incoming plasma, although our analysis, as Whipple's law itself, would not hold.

Our correction to Whipple's law, given by Eqs. (31), (33)-(35), depends on $M \theta$ and the temperature ratio $\beta$ $\equiv T_{i} / T_{e}$, in addition to both $M$ and retarding bias $V_{P}$. For typical values $\beta=O(1)$, perturbations outside the sheath, which are steady in the spacecraft frame, decay monotonically away from the RPA. The perturbed potential is positive, as the rejected-ion space charge itself, and results in opposite, competing effects on the current: Incoming ions are subject to a retarding decceleration, but once in the sheath they face a reduced potential hill in coming to the retarding grid. For $\alpha_{E} \sim 1$ and $\beta=0.5-1$, the correction to the current amounts to $15 \%-30 \%$, being negative at $M \theta=0$ but positive at $M \theta=2$. From the extremum in the slope of the current characteristic $I\left(V_{P}\right)$, sometimes used for a quick estimate of $T_{i}$, the corrected $T_{i}$ is only $5 \%-15 \%$ larger (smaller) than Whipple's value for $M \theta=0$ (for $M \theta=2$ ).

Our linear analysis can be extended to any ionospheric ion distribution that is Vlasov-stable against quasineutral ion-acoustic waves. Actually, the full steady solution, involving the beam of rejected ions, might itself be unstable against Lower Hybrid perturbations. These need extend, however, over extremely long distances along the geomagnetic field, which is only possible in the special case of a spacecraft moving nearly parallel to the field. ${ }^{20}$ 


\section{ACKNOWLEDGMENT}

This work was supported by the Comisión Interministerial de Ciencia y Tecnología of Spain, under Grant No. PB97-0574-C04-1.

\section{APPENDIX: UNIQUENESS OF SOLUTION}

We show here that in Eq. (25), written as

$$
\begin{aligned}
\frac{e \widetilde{V}_{1}\left(x, \bar{k}_{\perp}\right)}{k_{B} T_{i}}= & \int_{-\infty}^{\infty} \frac{d k_{x}}{2 \pi} \frac{\exp \left(i k_{x} x\right)}{\beta+L(\bar{k})}\left[\int_{-\infty}^{0} d x^{\prime} e^{-i k_{x} x^{\prime}}\right. \\
& \left.+\int_{0}^{\infty} d x^{\prime} e^{-i k_{x} x^{\prime}}\right] \frac{\widetilde{N}_{1}^{-}\left(x^{\prime}, \bar{k}_{\perp}\right)}{N_{\infty}},
\end{aligned}
$$

the contribution from the $x^{\prime}>0$ range of integration vanishes for $x<0$ whatever the function $\tilde{N}_{1}^{-}$, so that our choice of right-hand-side in (24) for $x>0$ had no effect on the resulting solution in the physical domain of definition. The vanishing of the $x^{\prime}>0$ contribution to (A1) for $x<0$ is a result of the property

$$
\int_{-\infty}^{\infty} \frac{d k_{x}}{2 \pi} \frac{\exp \left[-i k_{x}\left(x^{\prime}-x\right)\right]}{\beta+L\left(k_{x}, \bar{k}_{\perp}\right)} \equiv 0, \quad x^{\prime}-x>0,
$$

which arises from facts (i) $L\left(k_{x}\right)$, when continued analytically for complex $k_{x}$, is analytical in the lower half-plane, and (ii) the equation $\beta+L\left(k_{x}\right)=0$ has no roots in that halfplane. Then the integral (A2), rewritten as

$$
\begin{aligned}
& \frac{\delta\left(x^{\prime}-x\right)}{\beta+L_{-}(\infty)}+\int_{-\infty}^{\infty} \frac{d k_{x}}{2 \pi} \frac{\left[L_{-}(\infty)-L\left(k_{x}\right)\right] \exp \left[-i k_{x}\left(x^{\prime}-x\right)\right]}{\left[\beta+L_{-}(\infty)\right]\left[\beta+L\left(k_{x}\right)\right]}, \\
& \quad x^{\prime}-x>0,
\end{aligned}
$$

will clearly vanish; here, $L_{-}(\infty) \equiv L\left(\left|k_{x}\right| \rightarrow \infty, \operatorname{Im} k_{x}<0\right)$.

To establish facts (i) and (ii) above, we note the relation of our function $L(\zeta)$ in Eq. (27) to the plasma dispersion function $Z(\zeta),{ }^{24}$

$$
\begin{aligned}
& L=- \frac{1}{2} d Z / d \zeta \\
& Z(\zeta) \equiv 2 i \int_{0}^{\infty} d \sigma \exp \left(-\sigma^{2}+2 i \sigma \zeta\right) \\
& {\left[=\int_{-\infty}^{\infty} \frac{d t}{\sqrt{\pi}} \frac{\exp \left(-t^{2}\right)}{t-\zeta} \text { for } \operatorname{Im} \zeta>0\right], }
\end{aligned}
$$

with the large- $\zeta$ asymptotic approximation, $L(\zeta) \sim-\frac{1}{2} \zeta^{2}$, which was used in Sec. IV. We also note that $d Z(\zeta) / d \zeta$ is an entire function of $\zeta$, and is equal to a positive number nowhere in the upper half-plane $\operatorname{Im} \zeta>0$, there existing therefore no $\operatorname{Im} k_{x}<0$ solution to the equation $\beta+L\left(k_{x}\right)=0$, as advanced. Finally, note that this equation is the dispersion relation for electrostatic waves of phase velocity much less than the electron thermal speed of a Maxwellian plasma at rest $^{24}$

$$
\frac{\lambda_{\mathrm{Di}}^{2}}{\lambda_{\mathrm{De}}^{2}}-\frac{1}{2} \frac{d Z}{d \zeta}=-k^{2} \lambda_{\mathrm{Di}}^{2} \approx 0,
$$

when taking long wave numbers $\left(k \ll 1 / \lambda_{\text {De }}, 1 / \lambda_{\text {Di }}\right)$, with $\zeta$ in (27) standing for $\omega / k\left(2 k_{B} T_{i} / m_{i}\right)^{1 / 2}$. Property (A2) relates then to the fact that a Maxwellian plasma is stable in the Vlasov sense against quasineutral ion-acoustic waves. This shows that a solution to the linearized RPA problem can be determned for any non-Maxwellian ionospheric plasma that is stable against such waves.

${ }^{1}$ K. H. Wright, N. H. Stone, J. Sorensen, J. D. Winningham, and C. Gurgiolo, Geophys. Res. Lett. 25, 417 (1998).

${ }^{2}$ V. V. Skvortsov, L. V. Nosachev, and E. M. Netsvetailov, Cosmic Res. 7, 373 (1969).

${ }^{3}$ G. Fournier, Phys. Lett. A 34, 241 (1971).

${ }^{4}$ K. Spenner, W. C. Knudsen, and W. Lotze, J. Geophys. Res. 100, 14999 (1995).

${ }^{5}$ K. I. Gringauz, in Solar Terrestrial Physics, edited by J. W. King and W. S. Newman (Academic, New York, 1967); S. J. Bauer and A. F. Nagy, Proc. IEEE 63, 230 (1975); I. H. Hutchinson, Principles of Plasma Diagnostics (Cambridge University Press, Cambridge, UK, 1987), Chap. 3; M. C. Kelley, The Earth's Ionosphere (Academic, San Diego, CA, 1989), App. A.

${ }^{6}$ C. K. Cho and S.-Y. Su, Adv. Space Res. 23, 1537 (1999).

${ }^{7}$ S. Minami and Y. Takeya, J. Geophys. Res. 87, 713 (1982).

${ }^{8}$ D. I. Reasoner, S. D. Shawhan, and G. Murphy, J. Geophys. Res. 91, 13463 (1986).

${ }^{9}$ J. E. Sorensen, N. H. Stone, and K. H. Wright, Jr., J. Geophys. Res. 102, 24117 (1997).

${ }^{10}$ W. C. Knudsen, J. Bakke, K. Spenner, and V. Novak, Space Sci. Instrum. 4, 351 (1979).

${ }^{11}$ B. L. Cragin, W. B. Hanson, and S. Sanatani, J. Geophys. Res. 87, 4395 (1982).

${ }^{12}$ G. P. Mantas and W. B. Hanson, J. Geophys. Res. 92, 8559 (1987)

${ }^{13}$ E. C. Whipple, Proc. IRE 47, 2023 (1959).

${ }^{14}$ J. H. Hoffman, Proc. IEEE 57, 1063 (1969); S. Chandra, B. E. Troy, Jr., J. L. Donley, and R. E. Bourdeau, J. Geophys. Res. 75, 3867 (1970); W. J. Raitt, J. Blades, T. S. Bowling, and A. P. Willmore, J. Phys. E 6, 443 (1973).

${ }^{15}$ W. C. Knudsen, J. Geophys. Res. 71, 4669 (1966); W. B. Hanson, D. R. Frame, and J. E. Midgley, ibid. 77, 1914 (1972); P. D. Goldan, E. J. Yadlowsky, and E. C. Whipple, Jr., ibid. 78, 2907 (1973).

${ }^{16}$ C. K. Chao and S.-Y. Su, Phys. Plasmas 7, 101 (2000).

${ }^{17}$ L. W. Parker and E. C. Whipple, J. Geophys. Res. 75, 4720 (1970).

${ }^{18}$ E. C. Whipple, Jr., J. M. Warnock, and R. H. Winkler, J. Geophys. Res. 79, 179 (1974).

${ }^{19}$ B. E. Troy, Jr. and E. J. Maier, J. Geophys. Res. 80, 2236 (1975).

${ }^{20}$ B. Hanson and B. L. Cragin, J. Geophys. Res. 86, 10022 (1981).

${ }^{21}$ J. R. Sanmartín, Space Res. XIII, 497 (1973).

${ }^{22}$ N. Singh and W. C. Leung, Geophys. Res. Lett. 25, 741 (1998).

${ }^{23} \mathrm{H}$. Frisch, in Modern Mathematical Methods in Transport Theory, edited by W. Greenberg and J. Polewczak (Birkhäuser, Basel, 1991).

${ }^{24}$ T. H. Stix, Waves in Plasmas (American Institute of Physics, New York, 1998), Chap. 8. 